\title{
Kinetic helicity and MHD turbulence
}

\author{
SEAN OUGHTON and ROSSELLA PRANDI \\ Department of Mathematics, University College London, \\ Gower Street, London WC1E 6BT, UK
}

(Received 3 January 2000)

\begin{abstract}
The issue of dynamical anisotropy in helical three-dimensional magnetohydrodynamic turbulence with a mean magnetic field $\mathbf{B}_{0}$ is investigated. Using high-resolution direct numerical simulations, we follow the evolution of various isotropic initial states characterized by their different values of the kinetic helicity. The cross helicity and magnetic helicity of the initial conditions are also varied. In agreement with earlier work, we find that such initial states become anisotropic in of order an eddy-turnover time, with correlation lengths parallel to $\mathbf{B}_{0}$ remaining largely unchanged while finer scales are excited in the perpendicular directions. Moreover, it is found that the development of both the anisotropy and the energy are essentially independent of the initial level of kinetic helicity. The physics associated with this latter feature is discussed.
\end{abstract}

\section{Introduction}

Kinetic helicity $H_{k}=\frac{1}{2}\langle\mathbf{v} \cdot \boldsymbol{\omega}\rangle$, is a measure of the linkage and twist of the vorticity in a fluid (see Sec. 2 for definitions), and has long been recognized as a concept of importance in understanding and characterizing electrically conducting turbulent flows. The generation of magnetic fields by dynamo action, for example in the interiors of stars and planets, is thought to depend crucially on the presence of kinetic helicity. Indeed, pioneering work on the $\alpha$ effect found that a lack of reflectional symmetry in the underlying velocity field, associated with non-zero $H_{k}$, is a necessary condition for the existence of a kinematic dynamo (Steenbeck et al. 1966; Pouquet et al. 1976; Moffatt 1978; Krause and Rädler 1980). Moreover, in many space physics and astrophysical systems, significant levels of kinetic helicity are observed or inferred to exist, often as a consequence of (differential) rotation. It is therefore of some interest and importance to elucidate the role of $H_{k}$ in plasmas.

Quite generally, helicities can be associated with the linkage and/or twist of the field lines of the fields of interest (Moffatt 1969; Berger and Field 1984). In addition, there is a connection with the handedness of a vector field, with the latter often being restated in terms of a helicity; the presence of kinetic helicity, for example, implies that the velocity field lacks reflectional invariance. Care is required when considering these two aspects, however, since they are not equivalent (Frisch 1995). On the measurement side, the extraction of various helicities, and their spectra, from spacecraft datasets is also relatively easy to accomplish (Matthaeus et al. 1982,1986 ).

Here, we examine the influence of kinetic helicity on the dynamical development of three-dimensional magnetohydrodynamic (MHD) turbulence with a mean mag- 
netic field $\mathbf{B}_{0}$. A particular focus is the impact of $H_{k}$ on the development of spectral anisotropy. As is well known, a large value of kinetic helicity corresponds to a strong alignment of the velocity and vorticity fields. In the case of a non-conducting fluid, such alignments reduce the nonlinearity of the Navier-Stokes equation, and hence suppress the turbulent (inertial) transfer of energy. For a conducting flow, however, the Lorentz force provides a second source of nonlinearity. Thus, one might expect significant kinetic helicity to reduce the strength of the nonlinear MHD couplings, but with the subsequent impact being less than in an analogous Navier-Stokes situation. We show here that this is not necessarily the case, even for very high initial values of $H_{k}$. In particular, the evolution of energy and the development of spectral anisotropy appear to be largely independent of the initial level of kinetic helicity.

Previous work related to the topic considered here has mostly focused on the $B_{0}=0$ situation. This includes direct simulation studies with maximum helicity (kinetic and/or magnetic) initial conditions (Pouquet and Patterson 1978), eddydamped quasinormal Marbovian (EDQNM) simulations (Frisch et al. 1975; Pouquet et al. 1976), and a wide range of dynamo studies (e.g. Soward and Roberts 1992; Hughes et al. 1996).

The remainder of the paper is organized as follows. Section 2 introduces the governing equations and definitions for the relevant physical quantities and parameters. The numerical method employed is described in Sec. 3, with the results discussed in Sec. 4. The final section summarizes our conclusions.

\section{Governing equations and definitions}

The zero-mean turbulent velocity (v) and magnetic field (b) fluctuations occurring in an incompressible unforced magnetofluid, with a uniform (and static) external magnetic field $\mathbf{B}_{0}$ also present, are assumed to evolve according to the standard equations of incompressible three-dimensional MHD:

$$
\begin{gathered}
\frac{\partial \mathbf{v}}{\partial t}+\mathbf{v} \cdot \nabla \mathbf{v}=-\nabla p^{*}+\mathbf{b} \cdot \nabla \mathbf{b}+\mathbf{B}_{0} \cdot \nabla \mathbf{b}+\nu \nabla^{2} \mathbf{v}, \\
\frac{\partial \mathbf{b}}{\partial t}+\mathbf{v} \cdot \nabla \mathbf{b}=\mathbf{b} \cdot \nabla \mathbf{v}+\mathbf{B}_{0} \cdot \nabla \mathbf{v}+\eta \nabla^{2} \mathbf{b}, \\
\nabla \cdot \mathbf{v}=0, \quad \boldsymbol{\nabla} \cdot \mathbf{b}=0 .
\end{gathered}
$$

Lengths are normalized on $L$, where the computational box is a cube of side $2 \pi L$, while $\mathbf{B}_{0}, \mathbf{b}$ (in Alfvén units), and $\mathbf{v}$ are measured relative to an arbitrary speed $U_{0}$. Taking $L$ and $U_{0}$ equal to unity, the unit of time, $L / U_{0}$, then corresponds to one large-scale eddy-turnover time, provided that the total turbulent energy is also chosen to be unity, as is done hereinafter. In deriving these equations, the plasma density has been taken constant, and thus the total (mechanical plus magnetic) pressure $p^{*}$ is not an independent dynamical variable. In view of the solenoidal nature of $\mathbf{v}$ and $\mathbf{b}$, a magnetic vector potential $\mathbf{a}$ and a fluid stream function $\boldsymbol{\psi}$ can be introduced as usual $(\mathbf{b}=\boldsymbol{\nabla} \times \mathbf{a}, \mathbf{v}=\boldsymbol{\nabla} \times \boldsymbol{\psi})$. Recall also that, in these dimensionless units, the electric current $\mathbf{j}$ and the fluid vorticity $\boldsymbol{\omega}$ are respectively defined as $\mathbf{j}=\boldsymbol{\nabla} \times \mathbf{b}$ (the displacement current being neglected in MHD) and $\boldsymbol{\omega}=\boldsymbol{\nabla} \times \mathbf{v}$.

As is well known, the MHD equations are invariant under Galilean transformations, so that we take the mean velocity equal to zero, unlike the mean magnetic field $\mathbf{B}_{0}=B_{0} \hat{\mathbf{z}}$, which cannot be transformed away. Collisional dissipation is included in 
the model via isotropic viscous and resistive terms, whose dimensionless scalar coefficients $\nu$ and $\eta$ are the inverse (kinetic and magnetic) Reynolds numbers. In real plasmas, these transport coefficients take on a wide range of values. For example, the magnetic Reynolds number can be of order $10^{7}$ for fusion devices and of order $10^{12}$ in some astrophysical plasmas. Similarly, the magnetic Prandtl number $\nu / \eta$ is of order $10^{-7}$ in the Sun's photosphere but of order $10^{10}$ in the corona. In direct numerical simulations of a turbulent plasma, values for $\nu$ and $\eta$ are constrained by the maximum resolution that it is practical to use on the available computing systems. Here we take $\eta=\nu$ and use values of $\frac{1}{200}$ or $\frac{1}{400}$. The impact of anisotropic tensor viscosity is discussed elsewhere (e.g. Montgomery 1992; Oughton 1996, 1997).

In the ideal (i.e. $\nu=\eta=0$ ) continuum limit, $(2.1)$ conserve an infinite number of invariants and the ideal evolution is constrained by all these quantities. However, when even a small amount of dissipation is present, only the linear and quadratic invariants still play a key role (Kraichnan 1973). This follows since the invariance of higher-order quantities requires the existence of excitation at arbitrarily small scales. However, such scales are dynamically smoothed by the dissipative terms. Similarly, since numerical simulations are inherently restricted to a finite set of lengthscales, they too can only conserve at most the linear and quadratic invariants - completely independent of the presence or absence of numerical dissipation. For 3D MHD, the quadratic invariants - termed 'rugged invariants' - are the total energy $E=\frac{1}{2}\left\langle\mathbf{v}^{2}+\mathbf{b}^{2}\right\rangle \equiv E^{v}+E^{b}$, the magnetic helicity $H_{m}=\frac{1}{2}\langle\mathbf{a} \cdot \mathbf{b}\rangle$, and the cross helicity $H_{c}=\frac{1}{2}\langle\mathbf{v} \cdot \mathbf{b}\rangle$ (see e.g. Frisch et al. 1975). Although the kinetic helicity $H_{k}=$ $\frac{1}{2}\langle\mathbf{v} \cdot \boldsymbol{\omega}\rangle$ is a rugged invariant for $3 \mathrm{D}$ Euler flows, it is not one for MHD systems. It is nonetheless an important quantity, as noted in Sec. 1. Other bulk quantities useful in characterizing the turbulent dynamics, although again not invariants of the ideal MHD equations, are the enstrophy $\Omega=\frac{1}{2}\left\langle\boldsymbol{\omega}^{2}\right\rangle$, the mean square current density $J=$ $\frac{1}{2}\left\langle\mathbf{j}^{2}\right\rangle$, and the current helicity $H_{j}=\frac{1}{2}\langle\mathbf{b} \cdot \mathbf{j}\rangle$. Angle brackets denote volume averaging.

When analysing the simulation results, various normalized versions of the above parameters are often relevant. These include the normalized cross helicity $\sigma_{c}=$ $2 H_{c} / E$ and its spectral form $\sigma_{c}(k)=2 H_{c}(k) / E(k)$, the normalized magnetic helicity spectrum $\sigma_{m}(k)=k H_{m}(k) / E^{b}(k)$, the normalized kinetic helicity spectrum $\sigma_{k}(k)=H_{k}(k) /\left[k E^{v}(k)\right]$, and the normalized energy difference $\sigma_{D}=\left[E^{v}(k)-\right.$ $\left.E^{b}(k)\right] /\left[E^{v}(k)+E^{b}(k)\right]$. The dynamical information contained in these normalized values is complemented by the geometric 'mean alignment angle' content of ratios associated with Schwartz inequalities. For example, the globally averaged angle between the velocity and vorticity fields, $\Theta_{k}$, satisfies $\cos \Theta_{k}=\langle\mathbf{v} \cdot \boldsymbol{\omega}\rangle / \sqrt{\left\langle\mathbf{v}^{2}\right\rangle\left\langle\boldsymbol{\omega}^{2}\right\rangle} \equiv$ $H_{k} / \sqrt{\Omega E^{v}}$. An alternative interpretation is that $\cos \Theta_{k}$ is the correlation coefficient for the two fields. All of the normalized quantities defined in this paragraph are bounded by \pm 1 .

Note that magnetic helicity is not conserved when a mean magnetic field is present (Matthaeus and Goldstein 1982; Stribling et al. 1994, 1995), a feature that has recently provoked interest (Berger 1996, 1997; Matthaeus 1999; Montgomery and Bates 1999).

Using the above equations, one can obtain an evolution equation for $\mathbf{v} \cdot \boldsymbol{\omega}$ :

$$
\begin{aligned}
\left(\frac{\partial}{\partial t}+\mathbf{v} \cdot \nabla-\nu \nabla^{2}\right)(\mathbf{v} \cdot \boldsymbol{\omega})= & -\boldsymbol{\nabla} \cdot\left[\left(p-\frac{1}{2} v^{2}\right) \boldsymbol{\omega}\right] \\
& +\boldsymbol{\omega} \cdot \mathbf{j} \times \mathbf{B}+\mathbf{v} \cdot \boldsymbol{\nabla} \times(\mathbf{j} \times \mathbf{B})-2 \nu \frac{\partial v_{\alpha}}{\partial x_{\beta}} \frac{\partial \omega_{\alpha}}{\partial x_{\beta}},
\end{aligned}
$$


where $\mathbf{B}=\mathbf{B}_{0}+\mathbf{b}$. Integrating over an appropriate volume then gives an evolution equation for the kinetic helicity. Clearly this is a rather complicated equation, driven by both velocity-based and magnetically based nonlinearities, where the latter are responsible for the non-conservation of $H_{k}$ (in the ideal limit). The advective and dissipative terms are readily identified in (2.2), and the remaining terms alter $\mathbf{v} \cdot \boldsymbol{\omega}$ via distinct processes. Evidently the strong alignment of either the vorticity and the Lorentz force, or the velocity and the curl of the Lorentz force, is likely to produce a sizable change in $\mathbf{v} \cdot \boldsymbol{\omega}$. Note that a flow that is instantaneously nonhelical and/or irrotational will not remain so if $\nabla \times(\mathbf{j} \times \mathbf{B})$ has a non-zero projection on the velocity.

Finally in this section, we comment on the incompressibility assumption. The theory of nearly incompressible (NI) MHD (Zank and Matthaeus 1992) indicates that even in systems that are manifestly compressible, such as the solar wind, the small-scale fluctuations are nonetheless approximately incompressible when, for example, the plasma $\beta$ (ratio of thermal to magnetic pressure) is low, and the initial fluctuations are not dominated by compressive elements (Zank and Matthaeus 1992). Indeed, in such situations, the leading-order equations governing the fluctuations are precisely the equations of incompressible MHD. Observational, simulation, and modelling support for the quality of this approximation is well documented (see e.g. Tu and Marsch 1995; Matthaeus et al. 1996, 1998, 1999). Thus, the incompressible results presented below are also likely to be of relevance to various compressible space physics and astrophysical systems.

\section{Numerical method and initial conditions}

In order to investigate the development of anisotropy in helical flows, we numerically integrate the MHD equations (2.1) in a cube with periodic boundary conditions using a dealiased Fourier-Galerkin spectral code (Orszag 1971; Gottlieb and Orszag 1977; Canuto et al. 1988). The equations are solved in Fourier space, on a discrete grid with $N$ modes for each spatial direction. The components of the retained wavenumbers thus range between $-\frac{1}{2} N+1$ and $\frac{1}{2} N$. Aliasing removal for the nonlinear terms is carried out by exploiting the ' $\frac{3}{2}$ rule' (Patterson and Orszag 1971; Canuto et al. 1988). Time-advancement is achieved by means of an explicit second-order Runge-Kutta scheme. Fields in Fourier space are denoted by the same symbol used for the $x$-space representation, with the $\mathbf{x}$ or $\mathbf{k}$ argument made explicit when ambiguity might result. Further details of the numerical method are available in Oughton et al. (1994).

Initial conditions (ICs) are generated isotropically in Fourier space, with the amplitudes of the velocity and magnetic field coefficients satisfying

$$
\frac{1}{2}|\mathbf{v}(\mathbf{k})|^{2}=\frac{1}{2}|\mathbf{b}(\mathbf{k})|^{2}=\frac{C}{1+\left(k / k_{0}\right)^{q}} .
$$

The constant $C$ is chosen so that the total turbulent energy is unity and equipartitioned between the magnetic and kinetic contributions. It follows that the initial spectrum has a power-law form at large wavenumbers $\left(k \gg k_{0}\right)$ and is flat in the opposite limit. All the runs discussed here are initialized using the values $q=\frac{11}{3}$ and $k_{0}=4$, with only modes such that $3 \leqslant k \leqslant 8$ being excited.

Clearly the assignment of the phases of $\mathbf{v}(\mathbf{k})$ and $\mathbf{b}(\mathbf{k})$ determines the spectra of the kinetic helicity, cross helicity, and magnetic helicity. More specifically, we write 
every Fourier component of the velocity field in terms of its poloidal and toroidal components:

$$
\mathbf{v}(\mathbf{k})=i \mathbf{k} \times \hat{\mathbf{z}} \psi_{1}(\mathbf{k})-\mathbf{k} \times(\mathbf{k} \times \hat{\mathbf{z}}) \frac{\psi_{2}(\mathbf{k})}{k}
$$

$\left(\hat{\mathbf{z}}\right.$ is the unit vector parallel to $\left.\mathbf{B}_{0}\right)$. The form for $\mathbf{b}(\mathbf{k})$ is identical, with the potentials denoted by $a_{1}$ and $a_{2}$. In the case of completely uncorrelated initial conditions, each of the scalar potentials $a_{1}, a_{2}, \psi_{1}$, and $\psi_{2}$ is assigned using two independent Gaussian random variables, one each for the real and imaginary parts. Conversely, non-zero correlations between the potentials lead to non-zero levels of the various helicities. In particular, specifying the degree of correlation between $\psi_{1}$ and $\psi_{2}$ determines the value of the kinetic helicity. Similarly the $\psi_{1}-a_{1}$ and $\psi_{2}-a_{2}$ correlations control the cross-helicity level.

\section{Results and discussion}

Using initial conditions generated as described above, we now proceed to investigate the sensitivity of the turbulent evolution to the initial kinetic helicity level. For each distinct initial condition, various runs are typically performed, with the only parameter varied being the strength of $B_{0}$. Here we report on six such runsets, with each runset defined by its common initial condition (Table 1). For example the 'A' runs have (approximately) zero cross helicity and magnetic helicity, but a moderate value of kinetic helicity, while run $\mathrm{E}$ is similar but with a moderate value of $\sigma_{c}$. Note that the (initial) magnetic helicity is approximately zero in all runsets except F. The slightly anomalous nature of runs BB and FF is discussed in the table footnote.

\subsection{Evolution of global quantities}

An overview of the evolution of several typical runs is provided by Fig. 1, which displays time histories of the fluctuation energy, total rate of dissipation $\eta J+\nu \Omega$, normalized kinetic helicity, and normalized cross helicity $\sigma_{c}$. With one exception, the runs shown were all performed with $B_{0}=1$, and initial conditions with small or moderate cross helicity and moderate to large kinetic helicity (Table 1). The exception is a reference run with $B_{0}=0$ and moderate $H_{k}$ (run A0).

Figures $1(a, b)$ indicate that, as far as energy decay is concerned, the initial level of $H_{k}$ is of only minor importance. Indeed, the A1, B1, and D runs - for which $H_{k}$ is the only global parameter varied - are visually almost indistinguishable (solid lines) in these two plots. Moreover, runs C1 and E, which have a moderate level of $H_{k}$ but moderate and approximately equal cross-helicities, also exhibit very similar energy evolution and dissipation rate behaviour. Evidently the turbulence, in the process of establishing its desired nonlinear couplings, rapidly dispenses with the $\mathbf{v}-\boldsymbol{\omega}$ correlations imposed initially, thereby making the initial value of $H_{k}$ almost irrelevant in this context. Note that for $t \lesssim \frac{1}{2}$, all the $B_{0}=1$ curves shown in Figs $1(a, b)$ are indistinguishable. Recall that an eddy-turnover time is roughly the period required for the turbulence to establish an energy 'pipeline' from the large energy-containing scales to the small dissipative scales, via a nonlinear cascade. For freely decaying turbulence, one may extend the pipeline analogy by positing that, prior to $t \approx 1$, the pipeline is filling without being aware that a (dissipative) drain exists at its (high-wavenumber) end. In these cases, the peak dissipation rate occurs soon after the pipeline is filled. 
Table 1. Initial values for the run parameters.

\begin{tabular}{|c|c|c|c|c|c|c|}
\hline Run & $N$ & $\nu=\eta$ & $B_{0}$ & $\sigma_{c}$ & $\cos \Theta_{m}$ & $\cos \Theta_{k}$ \\
\hline A0 & 64 & $\frac{1}{200}$ & 0 & $\sim 0$ & $\sim 0$ & 0.42 \\
\hline Al & . & $\begin{array}{l}200 \\
.\end{array}$ & 1 & . & . & . \\
\hline $\mathrm{A} 2$ & . & . & 2 & . & . & . \\
\hline A3 & . & . & 4 & . & . & . \\
\hline $\mathrm{Bl}$ & . & . & 1 & . & . & 0.79 \\
\hline B2 & . & . & 2 & . & . & . \\
\hline B3 & . & & 4 & . & . & . \\
\hline $\mathrm{BB}$ & 128 & $\frac{1}{400}$ & 1 & . & . & . \\
\hline $\mathrm{D}$ & 64 & $\frac{1}{200}$ & 1 & . & . & 0.96 \\
\hline $\mathrm{Cl}$ & & . & 1 & 0.28 & . & $\sim 0$ \\
\hline $\mathrm{C} 2$ & . & . & 2 & . & . & . \\
\hline $\mathrm{E}$ & . & . & 1 & 0.31 & . & 0.42 \\
\hline $\mathrm{F}$ & . & & . & $\sim 0$ & 0.33 & . \\
\hline $\mathrm{FF}$ & 128 & $\frac{1}{400}$ & . & . & . & . \\
\hline
\end{tabular}

Blank lines separate sets of runs with the same initial conditions, differing only in the value for the d.c. magnetic field strength $B_{0}$. The two highest-resolution runs, BB and FF, have independently generated initial conditions but the same values of the global quantities characterizing their respective runsets. A dot indicates that the value is the same as that above it. All runs have initial kinetic and magnetic energy equal to one half.

It is also clear from Figs $1(a, b)$ that energy decay is significantly enhanced for $B_{0} \approx 0$ cases, as has been noted previously (see e.g. Shebalin et al. 1983; Oughton et al. 1994). This indicates that the decay of energy is reduced more effectively by a mean magnetic field - via suppression of parallel spectral transfer - than it is by significant levels of initial kinetic helicity (cf. Sec. 4.3).

Considering now Fig. 1(c), one sees that for $0<t \lesssim \frac{1}{4}, H_{k}$ typically decays rapidly. The dependence on the initial value is again weak, with $H_{k}$ being reduced by approximately $50 \%$ over this interval in each case shown. We suggest that this rapid early decorrelation of $\mathbf{v}$ and $\boldsymbol{\omega}$ is due to the action of the (strong) Lorentz force nonlinearities, which drive the velocity field without any particular correlation between $\psi_{1}$ and $\psi_{2}$ being imposed or enforced. For $\frac{1}{4} \lesssim t \lesssim 1.5$, there is a slower decrease of $H_{k}$, with a rough levelling out thereafter. The residual correlation associated with this levelling out is larger for larger initial values of $H_{k}$, although still relatively small. A striking feature of the plot is the collapse of the $H_{k}$ curves for runs Al, B1, and D onto each other. This suggests that for runs where the only parameter varied is the initial level of $H_{k}$, the evolution of $H_{k}$ is universal when normalized by the initial level.

The large oscillations in $H_{k}$ associated with run $\mathrm{Cl}$ are somewhat misleading, since $H_{k} \approx 0$ both initially and throughout the run. Note, however, that the period of the oscillations is the same as that characterizing Alfvénic fluctuations at the maximum allowed lengthscale, signifying that long-wavelength Alfvén waves are important in this case. As with the energy decay, the strongest overall decay of the kinetic helicity is associated with the $B_{0}=0$ case. In addition - and in contrast 

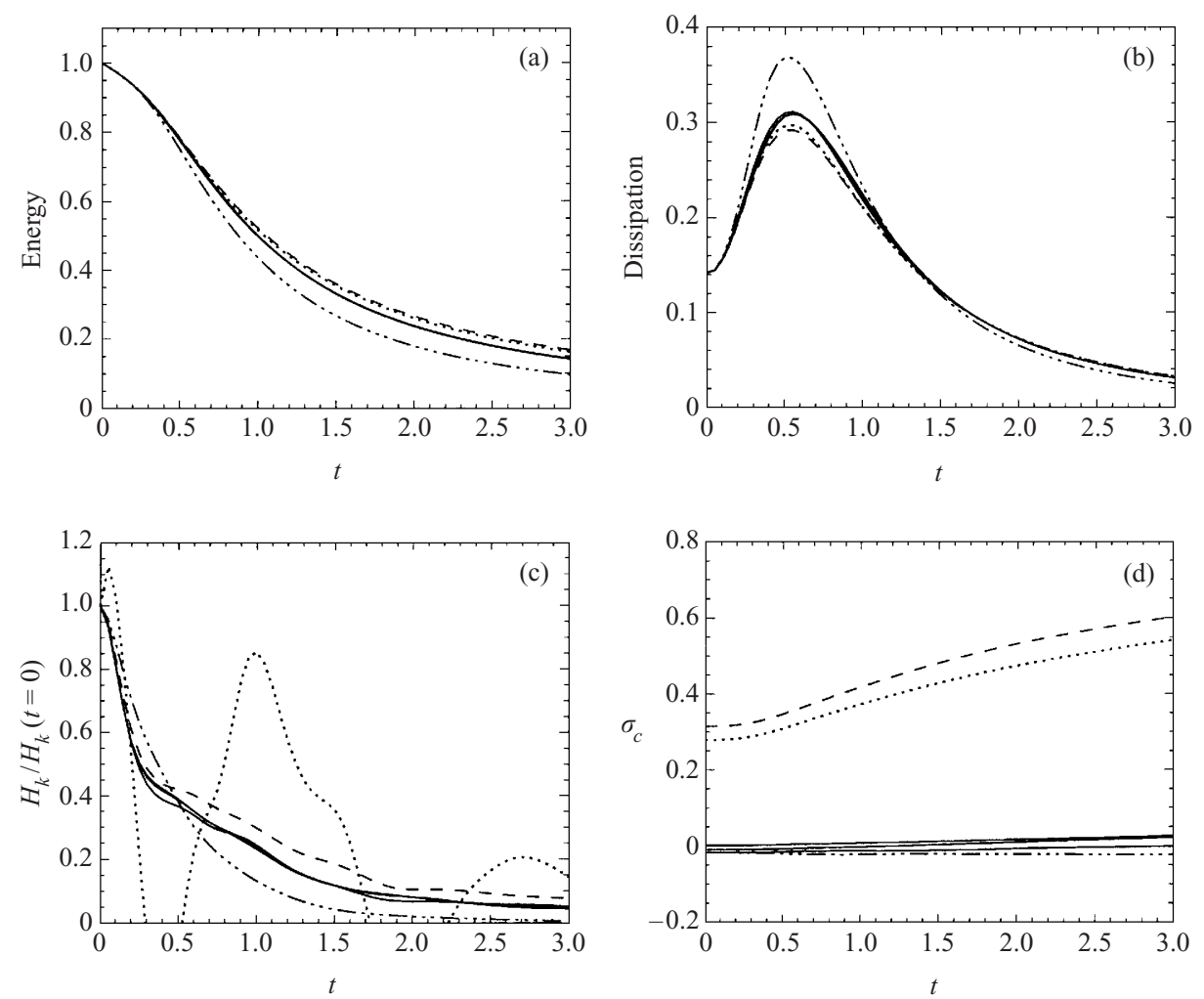

Figure 1. Time evolution curves for (a) fluctuation energy, (b) total (resistive plus viscous) dissipation rate, (c) kinetic helicity relative to the initial value, and (d) normalized cross-helicity Data are from runs A1, B1, D (solid curves), run C1 (dotted), and run E (dashed), which all have $B_{0}=1$, and run A0 (dash-dotted), which has $B_{0}=0$. See Table 1. Note the generally weak sensitivity of the various quantities to the initial value of $H_{k}$, and the rapid decrease of $H_{k}$.

to the non-zero $B_{0}$ cases - the decay of $H_{k}$ continues throughout the run, with no levelling out at later times. Evidently, the presence of a significant $B_{0}$ acts to preserve some 'residual' level of kinetic helicity. The mechanism is presumably related to the suppression of parallel spectral transfer discussed below, with the residual helicity being 'trapped' at relatively large (parallel) scales (cf. Fig. 2c).

Figure 1(d) displays the evolution of the normalized cross helicity for the various runs. As is well known, for turbulent MHD flows, $\sigma_{c}$ tends to increase in magnitude with time, via the dynamic alignment process (Dobrowolny et al. 1980; Matthaeus and Montgomery 1984; Grappin 1986; Pouquet et al. 1986). The simulation results shown suggest that, as for energy decay, the initial level of kinetic helicity is also largely irrelevant to the dynamic alignment process.

\subsection{Spectral evolution}

In this subsection, we examine the influence of helicity on the spectral evolution of some important quantities. Figure 2 displays various omnidirectional spectra from run FF at $t=0$ and four subsequent times. This $B_{0}=1$ run has moderate amounts of initial kinetic and magnetic helicity. Figure $2(a)$ shows the evolution 

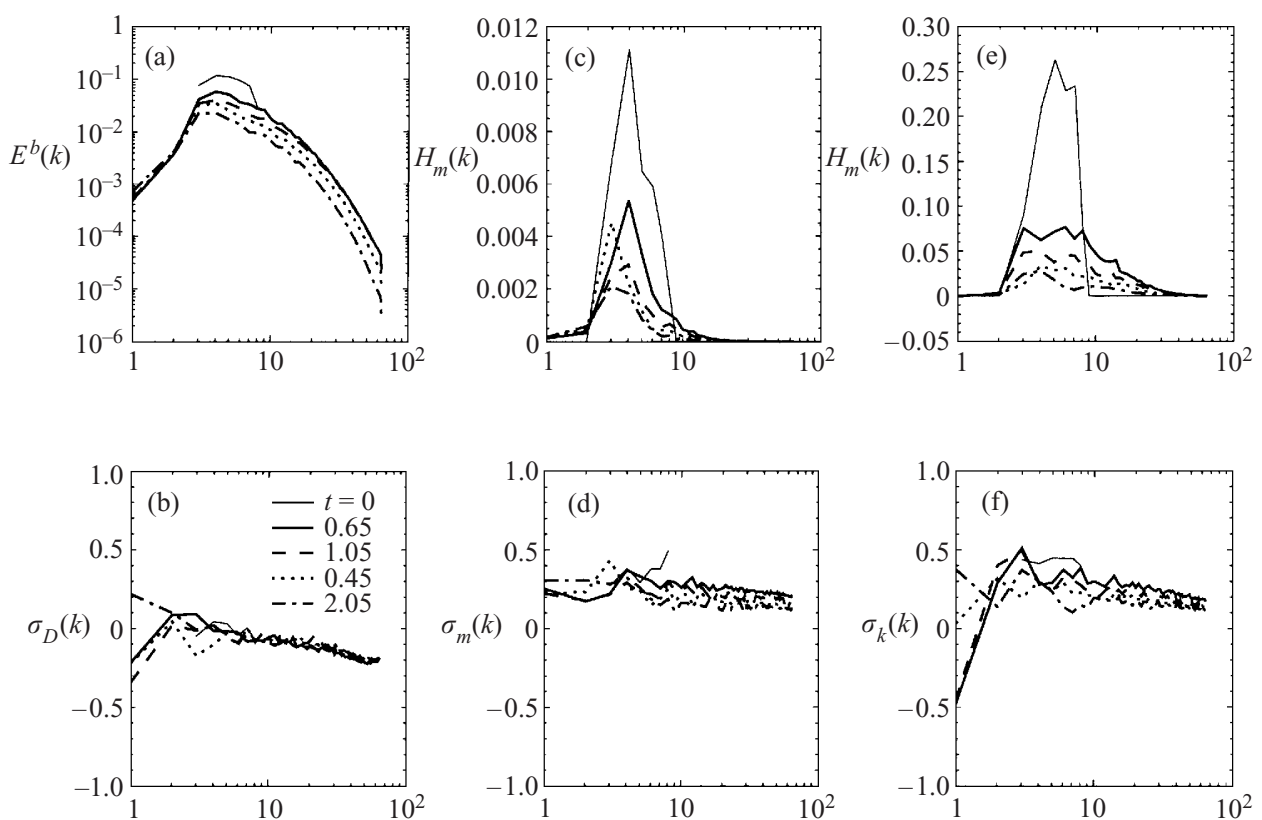

Figure 2. Omnidirectional wavenumber spectra at various times for run FF, which has $B_{0}=1$ and, initially, moderate $H_{k}$ and $H_{m}$.

of the magnetic energy spectra. It is clear that much of the decay is self-similar (Hossain et al. 1995; Galtier et al. 1997), even though there is no well-defined (powerlaw) inertial range present.

Also evident is a slight but progressive build-up of kinetic and magnetic energy at the (two) smallest wavenumbers. Almost all runs performed show this effect out to at least two eddy-turnover times, with the weakest build-up apparently being associated with $B_{0}=0$ runs. While this could be construed as evidence of a weak inverse cascade of magnetic energy, and thus dynamo action, it is worth recalling that the inverse cascade of magnetic energy is often associated with an inverse cascade of magnetic helicity when this latter quantity is an ideal invariant of the system (Frisch et al. 1975; Stribling and Matthaeus 1990, 1991). However, as noted in Sec. 2, when $B_{0} \neq 0, H_{m}$ is not an invariant, so that the origin of an inverse cascade process is uncertain. Nonetheless, Figs $2(\mathrm{c}, \mathrm{d})$ reveal that the magnetic helicity at the largest scales also tends to increase, both in an absolute and in a relative sense. This effect is less evident, although not absent, in runs with approximately zero initial magnetic helicity, and is the subject of current investigation. Note that for this run the peak of the magnetic helicity spectrum tends to move towards lower $k$ with time, which is also consistent with inverse cascade activity. However, the figure also clearly shows that significant magnetic helicity is transferred towards higher wavenumbers, where $\sigma_{m}(k)$ is very roughly flat, although decaying with time. (cf. Stribling et al. 1994, 1995).

In solar wind observations (Matthaeus and Goldstein 1982; Goldstein et al. 1994), and also in the simulations of Stribling et al. (1995), $\sigma_{m}(k)$ is found to fluctuate about zero, in marked contrast to the essentially single-signed spectra presented 
here. However, the results are not directly comparable. The Stribling et al. (1995) simulations are ideal, and the spectra obtained therein are averages over many tens or hundreds of eddy-turnover times. Our results are dissipative, and extend out to only a few eddy-turnover times, so that the initial bulk value of $H_{m}$ has not yet been sufficiently eroded to reveal the long-time fluctuations. The relationship between the $H_{m}$ evolution that we report on here and that in the solar wind remains to be determined.

The normalized energy difference spectra (Fig. 2b) indicate that the magnetic energy tends to become more predominant with increasing $k$. This structure is established in a fraction of an eddy-turnover time, remains approximately steady thereafter, and holds for all the $B_{0}=1$ runs. At first sight, this seems to be in contradiction to the predictions of the Alfvén effect. This posits that in a system with a strong $\mathbf{B}_{0}$, small-scale incompressible MHD fluctuations are well approximated as Alfvén waves, i.e. small-amplitude fluctuations about a background field (Kraichnan 1967; Pouquet et al. 1976). Consequently, these fluctuations should obey $E^{v}(k) \approx E^{b}(k)$, or equivalently $\sigma_{D}(k) \approx 0$. There are several factors to consider here. First, for the $B_{0}=1$ runs, the energy in the turbulence is comparable to, rather than much less than, the energy in the mean field, although for small-scale fluctuations this should be less relevant, not the reverse as is actually observed. Secondly, the above physical justification for the Alfvén effect fails to take into account the anisotropic dynamics engendered by a mean field, and in particular the suppression of parallel spectral transfer, which results in the evolution of the flow towards quasi-two-dimensional states (see Sec. 4.3). Fluctuations that are strictly two-dimensional relative to $\mathbf{B}_{0}$ are dynamically unaware of the mean field, and are thus not subject to a $B_{0}$-induced Alfvén effect; however, the fluctuating large-scale field may play an analogous role (Kraichnan 1967; Pouquet et al. 1976; Hossain et al. 1995). The fact that the smaller scales are less Alfvénic suggests that these scales are the most quasi-2D-like, and thus the most anisotropic, as is also indicated by the results of the next section. Simulations using initial conditions with no significant initial $H_{k}$ display the same behaviour (Oughton 1994; Matthaeus et al. 1996). On the other hand, on comparing runs with increasing values of $B_{0}$ (e.g. runset A), one finds similar quasi-steady behaviour of $\sigma_{D}(k)$, but with (i) the flatness of the spectrum becoming more pronounced, and (ii) equipartition at these scales being an improving approximation. Both of these features are in accord with the Alfvén-effect predictions.

Two other factors that may influence the development of 'excess' $E^{b}$ are local, in $k$-space, dynamo activity (Pouquet et al. 1976) and the current-sheetvorticity quadrupole structures associated with small-scale turbulent reconnection (Matthaeus and Lamkin 1986). Such excess magnetic energy has been seen in many other simulations (Pouquet et al. 1976; Pouquet and Patterson 1978; Shebalin et al. 1983; Matthaeus and Lamkin 1986; Oughton et al. 1994; Matthaeus et al. 1996), and is also a robust feature in the solar wind, where observations indicate that $\sigma_{D} \approx-\frac{1}{3}$ (Matthaeus and Goldstein 1982; Roberts et al. 1987a,b). One typically finds that in the solar wind, $E / B_{0}^{2} \approx 1$, which in our units corresponds roughly to simulations with $B_{0}=1$. Thus, the simulations are consistent with the proposal that solar wind fluctuations have an energetically significant component of quasi-2D fluctuations (Matthaeus et al. 1990).

On the basis of EDQNM calculations for isotropic MHD, Pouquet et al. (1976) suggested that, along with $\sigma_{D}(k) \approx 0$, the Alfvén effect should also be associated 
with a zero 'helicity difference' $\sigma_{H}(k)=H_{k}(k)-k^{2} H_{m}(k)$ at the smaller scales, or, more specifically $H_{k}(k)-H_{j}(k) \approx 0$ (Pouquet and Patterson 1978). Our simulations are in accord with this prediction, with $\sigma_{H}(k)$ typically having a magnitude much less than $\frac{1}{10}$, except at early times for some of the runs with larger initial helicity. Initial values of $\sigma_{H}(k)$ were in the range $0.3-0.6$.

Considering now the spectral evolution of the kinetic helicity, we see from Fig. 2 that $H_{k}(k)$ is predominantly forward-transferred, with an overall tendency to decay in amplitude. The other runs reported on here evince similar behaviour. This is reminiscent of the linear cascade of $H_{k}$ seen in Navier-Stokes turbulence (Brissaud et al. 1973), although, as (2.2) shows, the behaviour is more complicated because of Lorentz force influences.

On examining Fig. 2f, one observes that there is significant transfer of relative kinetic helicity to the largest scale. This is a typical feature of the simulations discussed here, as is the oscillation of $\sigma_{k}\left(k_{\min }\right)$. However, the oscillations do not appear to be dynamically significant, because of the small energy content of these modes. The similar oscillations seen in $\sigma_{D}\left(k_{\min }\right)$ suggest that both types of variations are associated with long-wavelength Alfvénic fluctuations. The figure also indicates that $H_{m}(k)$ and $H_{k}\left(k>k_{\min }\right)$ are positive at all times shown. This single-signed nature of the helicities, with the occasional exception at the largest scales, appears to be a robust result, provided only that the relative helicities are not approximately zero (however, see Stribling et al. (1995) for discussion of distinct behaviour at long times). It is an aspect of another robust feature of the evolution, namely that, for a given $k, H_{k}(k)$ and $H_{m}(k)$ have a very strong tendency to have the same sign, even if this is not the case initially. This is, of course, almost necessary for compatibility with the helicity difference prediction associated with the Alfvén effect discussed above. When the relative helicity levels are initially low at all values of $k$ excited, the turbulent fluctuations in $\sigma_{m}(k)$ or $\sigma_{k}(k)$ behave differently, with numerous sign changes taking place over time. Clearly, for such low initial levels, the turbulent fluctuations in these quantities are larger in amplitude than any average values.

In summary, the kinetic helicity does not appear to have a strong influence on the spectral (energy) dynamics of unforced turbulent MHD flows. In contrast, spectral evolution can be significantly influenced by cross-helicity and magnetic-helicity levels, as discussed elsewhere (Frisch et al. 1975; Pouquet et al. 1976; Dobrowolny et al. 1980; Matthaeus and Montgomery 1984; Grappin 1986; Oughton et al. 1994; Stribling et al. 1994, 1995).

\subsection{Anisotropy}

In agreement with the previous literature on the subject, we observe that when $B_{0}^{2} \gtrsim E$, any isotropic initial condition tends to evolve towards an anisotropic quasitwo-dimensional state (Shebalin et al. 1983; Carbone and Veltri 1990; Oughton et al. 1994, 1998; Matthaeus et al. 1996, 1998). At leading order, the dynamical process responsible for this development of anisotropy can be understood in terms of the nonlinear interaction of Alfvén waves (Shebalin et al. 1983). Examining the situation in Fourier space, one finds that energy is preferentially transferred towards higher $\mathbf{k}_{\perp}$ at fixed $\mathbf{k}_{\|}$, so that spectral transfer of energy parallel to $\mathbf{B}_{0}$ is (approximately) frozen out. In physical space, this manifests itself as flows that develop fine-scale structure perpendicular to the mean field, while maintaining essentially unchanged characteristic parallel lengthscales. Important refinements to this picture are discussed elsewhere (Grappin 1986; Kinney and McWilliams 1998; 

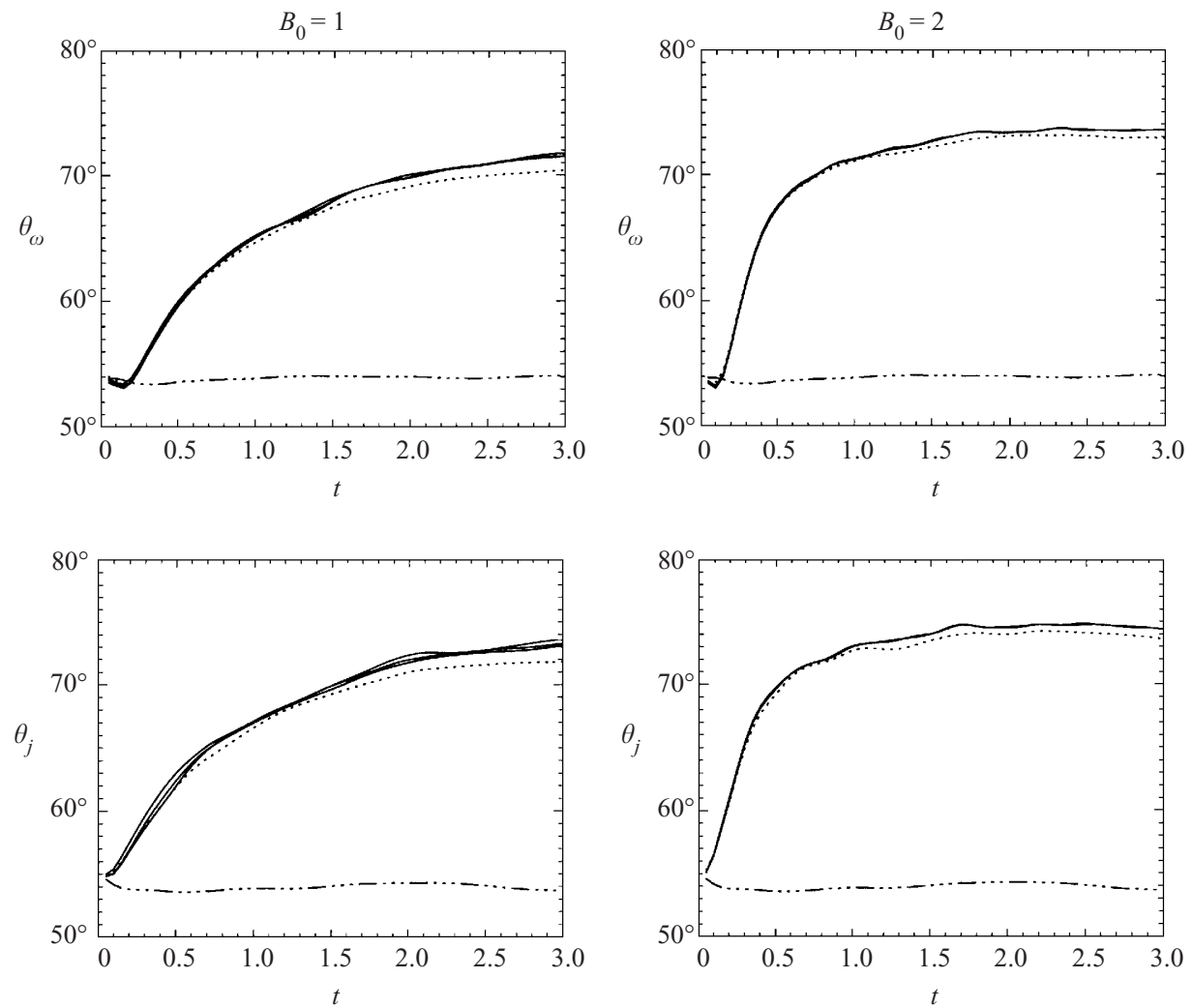

Figure 3. Anisotropy angles for the vorticity and current density as functions of time; see (4.1). We compare the effect that kinetic helicity and cross helicity have on the development of anisotropy. The left-hand side shows runs with $B_{0}=1$ : A1, B1, D (solid lines), and C1 (dotted). The right-hand side shows runs with $B_{0}=2$ : A2, B2 (solid), and C2 (dotted). The curves fluctuating about $54^{\circ}$ are for run $\mathrm{A} 0$, with $B_{0}=0$.

Matthaeus et al. 1998; Oughton et al. 1998). The net result is that after a few eddy-turnover times, the turbulence is in a state which is well characterized by descriptions based on reduced MHD (RMHD), even when the initial states are emphatically not RMHD-like (Strauss 1976; Montgomery 1982; Zank and Matthaeus 1992).

The degree of anisotropy can be measured quantitatively using a ratio of (suitably weighted) average lengthscales in the directions parallel and perpendicular to $\mathbf{B}_{0}$, or by its spectral counterpart, the anisotropy angle $\theta_{Q}$ (Shebalin et al. 1983),

$$
\tan ^{2} \theta_{Q}=\frac{\sum k_{\perp}^{2}|\mathbf{Q}(\mathbf{k}, t)|^{2}}{\sum k_{z}^{2}|\mathbf{Q}(\mathbf{k}, t)|^{2}},
$$

where the sums are over all wavevectors, $k_{\perp}^{2}=k_{x}^{2}+k_{y}^{2}$, and $\mathbf{Q}$ is any one among $\boldsymbol{\psi}, \mathbf{v}, \boldsymbol{\omega}, \mathbf{a}, \mathbf{b}, \mathbf{j}$. By definition, $\theta_{Q}$ ranges between $0^{\circ}$ and $90^{\circ}$ (respectively, excitation of only parallel modes and only perpendicular modes), taking on the value $\arctan \sqrt{2} \approx 54.74^{\circ}$ in isotropic conditions.

In Fig. 3, we plot anisotropy angles resulting from the analysis of the simulation data. Before discussing the effect of the initial value of $H_{k}$, we report on a few general features regarding the anisotropy, which are in agreement with the discus- 
sion presented in Oughton et al. (1994). Following an initial increase with time, the angles reach more or less saturated values after a few eddy-turnover times, which they retain for the remainder of the run(s). Both the time taken to attain the plateau and the value of the plateau itself depend on the intensity of $B_{0}$ and on the field $\mathbf{Q}$ under consideration. Furthermore, the usual ordering $\theta_{\psi, a}<\theta_{v, b}<\theta_{\omega, j}$ is seen, suggesting that the smaller scales are more anisotropic. Discussions on the scaling of anisotropy angles with the ratio of fluctuating to total magnetic field strength can be found in Matthaeus et al. (1998) and Oughton et al. (1998).

Let us now consider the influence of kinetic helicity on the development of spectral anisotropy. As can be seen in the left-hand panels of Fig. 3, the anisotropy angles for runs with $B_{0}=1$ and low (A1), moderate (B1), and high (D) initial kinetic helicity follow essentially the same curve. This is consistent with the insensitivity of the evolution of the energy, and other integral quantities, to the initial value of $H_{k}$ discussed above. Qualitatively similar features hold for higher values of the external magnetic field, but with stronger anisotropy being evident (right-hand panels). However, the increase in anisotropy with $B_{0}$ saturates for $B_{0} \gtrsim 3$, as is also the case for ICs with $H_{k} \approx 0$ (Oughton et al. 1994).

While the anisotropy evolution is relatively insensitive to the initial value of $H_{k}$, it is much more sensitive to the initial cross helicity. For comparison, we also plot data from run $\mathrm{Cl}$ (dotted), which has $\sigma_{c}(t=0) \approx 0.28$. This reveals that even relatively small $\mathbf{v}-\mathbf{b}$ correlations noticeably weaken the development of the anisotropies, although the effect is rather small. In fact, as is well known (see e.g. Dobrowolny et al. 1980; Grappin 1986), non-zero cross helicity is associated with reduced strength of the nonlinear couplings, and thus a reduction of spectral transfer. Since the development of the anisotropy is a nonlinear process (Shebalin et al. 1983; Oughton et al. 1994, 1998; Oughton 1996; Matthaeus et al. 1998), one then expects initial conditions with cross helicity to evolve into states that are less anisotropic than their zero- $H_{c}$ analogues, as is seen to be the case here.

\section{Conclusions}

We have presented results from direct numerical simulations of unforced incompressible three-dimensional MHD turbulence in a strong external magnetic field, which show that the presence of kinetic helicity does not strongly affect the development of the flows, in the sense that the evolution of energy and spectral anisotropy are almost independent of the initial level of kinetic helicity. These results have been obtained for the freely decaying case, with no energy or helicity supplied during the evolution, and are thus influenced by the fast decay rate of the kinetic helicity itself. We find that the turbulent dynamics rapidly destroys the initial correlation between the velocity and vorticity fields. This contrasts with the typical enhancement of the $\mathbf{v}-\mathbf{b}$ correlation (i.e. the cross helicity) via the dynamic alignment process (Dobrowolny 1980). A possible explanation for this dichotomy lies in the dimensionality of the contributing fields. Any velocity fluctuations that are strictly two-dimensional will of necessity have $\mathbf{v} \cdot \boldsymbol{\omega} \equiv 0$, whereas non-zero values of the cross-helicity can occur independent of the the two- or three-dimensional nature of $\mathbf{v}$ and $\mathbf{b}$. Since the anisotropy generation process is associated with evolution towards quasi-two-dimensional states, it is consistent that the kinetic helicity decreases apace with the increase in anisotropy angles. However, this cannot be a full explanation, since $H_{k}$ also decays rapidly in $B_{0}=0$ runs.

At variance with what is known to occur for Navier-Stokes turbulence (Brissaud 
et al. 1973; André and Lesieur 1977; Moffatt and Tsinober 1992), we have shown that the depletion of the (velocity-based) nonlinearity in the momentum equation (2.1a) does not significantly inhibit the spectral transfer of energy to small scales. The usual picture of high-Reynolds-number MHD flows as a complex mixture of waves and turbulence remains appropriate for flows with substantial initial kinetic helicity. This is true for all values of $\mathbf{B}_{0}$, although when $\mathbf{B}_{0}$ is not energetically weak relative to the turbulence, the energy flux is primarily to small perpendicular scales, with only weak transfer in the parallel direction, and an associated reduced decay of energy. In effect, the nonlinear process that produces the observed spectral anisotropy is almost immune to the initial value of $H_{k}$.

The physical basis for the insensitivity of the dynamics to $H_{k}$ can be seen by recalling that $\partial \mathbf{v} / \partial t \sim \mathbf{v} \times \boldsymbol{\omega}+\mathbf{j} \times \mathbf{b}$, where only the nonlinear terms are considered. It follows that the velocity will be strongly driven by the Lorentz force term, even when $\mathbf{v} \times \boldsymbol{\omega}$ is weak by virtue of the alignment of the two fields.

The caveat to the above conclusions regarding the irrelevance of the initial value of $H_{k}$ is that they may not hold when the $\mathbf{j} \times \mathbf{b}$ term is too weak to produce effective driving of $\mathbf{v}$ away from the initially helical states. Given that a broadband pool of magnetic fluctuations exists, $\mathbf{j} \times \mathbf{b}$ will be significant provided that $\mathbf{j}$ and $\mathbf{b}$ are not nearly parallel. This last condition may be rephrased in terms of the current helicity $H_{j}=\frac{1}{2}\langle\mathbf{b} \cdot \mathbf{j}\rangle$, where the requirement is then that $H_{j}$ is not near-extremal. When the magnetic helicity is dominated by contributions from a narrow range of wavenumbers centred on $k_{\mathrm{H}}$, one has $H_{j} \approx k_{\mathrm{H}}^{2} H_{m}$, and the condition for the insensitivity to initial values of $H_{k}$ is equivalent to demanding that $H_{m}$ is not near-extremal. Thus we conjecture that decaying flows that do not simultaneously have almost-extremal values of both the kinetic and the magnetic helicity will rapidly remove the initial $\mathbf{v}-\boldsymbol{\omega}$ correlation and proceed thereafter as if there was initially no significant kinetic helicity.

In summary, as far as the evolution of kinetic helicity is concerned, the natural action of unforced (and non-rotating) incompressible MHD turbulence appears to be to remove it from the flow, so that even a large initial value has little significance to the subsequent (energy-based) dynamics. Evidently, unless some process - such as rotation - acts to maintain, inject, or produce kinetic helicity, this quantity is rapidly disposed of via the turbulent dynamics.

\section{Acknowledgements}

Support for this work was provided by the UK PPARC under Grants GR/L63143 (R.P.) and GR/K98711 (S.O.). We thank M.A. Berger and W.H. Matthaeus for helpful discussions in connection with this work.

\section{References}

André, J. C. and Lesieur, M. 1977 Influence of helicity on high Reynolds number isotropic turbulence. J. Fluid Mech. 81, 187.

Berger, M. A. 1996 Inverse cascades in a periodic domain. Astrophys. Lett. Commun. 34, 225.

Berger, M. A. 1997 Magnetic helicity in a periodic domain. J. Geophys. Res. 102, 2637.

Berger, M. A. and Field, G. B. 1984 The topological properties of magnetic helicity. J. Fluid Mech. 147, 133.

Brissaud, A., Frisch, U., Léorat, J., Lesieur, M. and Mazure, A. 1973 Helicity cascades in fully developed isotropic turbulence. Phys. Fluids 16, 1366. 
Canuto, C., Hussaini, M. Y., Quarteroni, A. and Zang, T. A. 1988 Spectral Methods in Fluid Mechanics. Springer-Verlag, New York.

Carbone, V. and Veltri, P. 1990 A shell model for anisotropic magnetohydrodynamic turbulence. Geophys. Astrophys. Fluid Dyn. 52, 153.

Dobrowolny, M., Mangeney, A. and Veltri, P. 1980 Fully developed anisotropic hydromagnetic turbulence in interplanetary space. Phys. Rev. Lett. 45, 144.

Frisch, U. 1995 Turbulence. Cambridge University Press.

Frisch, U., Pouquet, A., Léorat, J. and Mazure, A. 1975 Possibility of an inverse cascade of magnetic helicity in magnetohydrodynamic turbulence. J. Fluid Mech. 68, 769.

Galtier, S., Politano, H. and Pouquet, A. 1997 Self-similar energy decay in magnetohydrodynamic turbulence. Phys. Rev. Lett. 79, 2807.

Goldstein, M. L., Roberts, D. A. and Fitch, C. A. 1994 Properties of the fluctuating magnetic helicity in the inertial and dissipation ranges of solar wind turbulence. J. Geophys. Res. 99. 11519

Gottlieb, D. and Orszag, S. A. 1977 Numerical Analysis of Spectral Methods: Theory and Applications. SIAM, Philadelphia.

Grappin, R. 1986 Onset and decay of two-dimensional magnetohydrodynamic turbulence with velocity-magnetic field correlation. Phys. Fluids $\mathbf{2 9}, 2433$.

Hossain, M., Gray, P. C., Pontius, D. H. Jr., Matthaeus, W. H. and Oughton, S. 1995 Phenomenology for the decay of energy-containing eddies in homogeneous MHD turbulence. Phys. Fluids 7, 2886.

Hughes, D. W., Cattaneo, F. and Kim, E.-J. 1996 Kinetic helicity, magnetic helicity and fast dynamo action. Phys. Lett. 223A, 167.

Kinney, R. and McWilliams, J. C. 1998 Turbulent cascades in anisotropic magnetohydrodynamics. Phys. Rev. E57, 7111.

Kraichnan, R. H. 1967 Inertial ranges in two-dimensional turbulence. Phys. Fluids 10, 1417.

Kraichnan, R. H. 1973 Helical turbulence and absolute equilibrium. J. Fluid Mech. 59, 745.

Krause, F. and Rädler, K.-H. 1980 Mean-Field Magnetohydrodynamics and Dynamo Theory. Akademie-Verlag, Berlin.

Matthaeus, W. H. 1999 Magnetic helicity and homogeneous turbulence models. In: Geophys ical Monograph 111. Proceedings of Magnetic Helicity in Space and Laboratory Plasmas (ed. M. R. Brown, R. C. Canfield and A. A. Pevtsov). AGU, Washington DC.

Matthaeus, W. H. and Goldstein, M. L. 1982 Measurement of the rugged invariants of magnetohydrodynamic turbulence in the solar wind. J. Geophys. Res. 87, 6011.

Matthaeus, W. H. and Lamkin, S. L. 1986 Turbulent magnetic reconnection. Phys. Fluids 29, 2513

Matthaeus, W. H. and Montgomery, D. 1984 Dynamic alignment and selective decay in MHD. In: Statistical Physics and Chaos in Fusion Plasmas (ed. C. W. J. Horton and L. E. Reichl), p. 285. New York, Wiley.

Matthaeus, W. H., Goldstein, M. L. and Smith, C. W. 1982 Evaluation of magnetic helicity in homogeneous turbulence. Phys. Rev. Lett. 48, 1256.

Matthaeus, W. H., Goldstein, M. L. and Lantz, S. R. 1986 The alpha dynamo parameter and measurability of helicities in magnetohydrodynamic turbulence. Phys. Fluids $\mathbf{2 9}, 1504$.

Matthaeus, W. H., Goldstein, M. L. and Roberts, D. A. 1990 Evidence for the presence of quasi-two-dimensional nearly incompressible fluctuations in the solar wind. J. Geophys. Res. 95, 20673.

Matthaeus, W. H., Ghosh, S., Oughton, S. and Roberts, D. A. 1996 Anisotropic threedimensional MHD turbulence. J. Geophys. Res. 101, 7619-7629.

Matthaeus, W. H., Oughton, S., Ghosh, S. and Hossain, M. 1998 Scaling of anisotropy in hydromagnetic turbulence. Phys. Rev. Lett. 81, 2056.

Matthaeus, W. H., Zank, G. P., Smith, C. W. and Oughton, S. 1999 Turbulence, spatial transport, and heating of the solar wind. Phys. Rev. Lett. 82, 3444

Moffatt, H. K. 1969 The degree of knottedness of tangled vortex lines. J. Fluid Mech. 35 117.

Moffatt, H. K. 1978 Magnetic Field Generation in Electrically Conducting Fluids. Cambridge University Press. 
Moffatt, H. K. and Tsinober, A. 1992 Helicity in laminar and turbulent flow. Annu. Rev. Fluid Mech. 24, 281.

Montgomery, D. C. 1982 Thresholds for the onset of fluid and magnetofluid turbulence. Physica Scripta T2/1, 506.

Montgomery, D. C. 1992 Modifications of magnetohydrodynamics as applied to the solar wind. J. Geophys. Res. 97, 4309.

Montgomery, D. C. and Bates, J. W. 1999 The geometry and symmetries of magnetohydrodynamic turbulence: anomalies of spatial periodicity. Phys. Plasmas 6, 2727.

Orszag, S. A. 1971 Numerical simulation of incompressible flows within simple boundaries: I. Galerkin (spectral) representations. Stud. Appl. Math. 50, 293.

Oughton, S. 1996 Ion parallel viscosity and anisotropy in MHD turbulence. J. Plasma Phys. 56, 641 .

Oughton, S. 1997 Energy dynamics in linear MHD with ion parallel viscosity. J. Plasma Phys. 58, 571 .

Oughton, S., Priest, E. R. and Matthaeus, W. H. 1994 The influence of a mean magnetic field on three-dimensional MHD turbulence. J. Fluid Mech. 280, 95.

Oughton, S., Ghosh, S. and Matthaeus, W. H. 1998 Scaling of spectral anisotropy with magnetic field strength in decaying MHD turbulence. Phys. Plasmas 5, 4235-4242.

Patterson, G. S. and Orszag, S. A. 1971 Spectral calculations of isotropic turbulence: Efficient removal of aliasing interactions. Phys. Fluids 14, 2538.

Pouquet, A. and Patterson, G. S. 1978 Numerical simulation of helical magnetohydrodynamic turbulence. J. Fluid Mech. 85, 305.

Pouquet, A., Frisch, U. and Léorat, J. 1976 Strong MHD helical turbulence and the nonlinear dynamo effect. J. Fluid Mech. 77, 321.

Pouquet, A., Meneguzzi, M. and Frisch, U. 1986 Growth of correlations in magnetohydrodynamic turbulence. Phys. Rev. A33, 4266.

Roberts, D. A., Klein, L. W., Goldstein, M. L. and Matthaeus, W. H. 1987a The nature and evolution of magnetohydrodynamic fluctuations in the solar wind: Voyager observations. J. Geophys. Res. 92, 11021.

Roberts, D. A., Goldstein, M. L., Klein, L. W. and Matthaeus, W. H. 1987b Origin and evolution of fluctuations in the solar wind: Helios observations and Helios-Voyager comparisons. J. Geophys. Res. 92, 12023.

Shebalin, J. V., Matthaeus, W. H. and Montgomery, D. 1983 Anisotropy in MHD turbulence due to a mean magnetic field. J. Plasma Phys. 29, 525.

Soward, A. M. and Roberts, P. H. 1992 Dynamo theory. Annu. Rev. Fluid Mech. 24, 459.

Steenbeck, M., Krause, F. and Rädler, K. H. 1966 Berechnung der mittleren LorentzFeldstärke für ein elektrisch leitendes Medium in turbulenter, durch Coriolis Kräfte beeinflußter Bewegung. Z. Naturforsch 21 a, 369.

Strauss, H. R. 1976 Nonlinear, three-dimensional magnetohydrodynamics of non-circular tokamaks. Phys. Fluids 19, 134.

Stribling, T. and Matthaeus, W. H. 1990 Statistical properties of ideal three-dimensional magnetohydrodynamics. Phys. Fluids B2, 1979.

Stribling, T. and Matthaeus, W. H. 1991 Relaxation processes in a low order threedimensional magnetohydrodynamics model. Phys. Fluids B3, 1848.

Stribling, T., Matthaeus, W. H. and Ghosh, S. 1994 Nonlinear decay of magnetic helicity in magnetohydrodynamics with a mean magnetic field. J. Geophys. Res. 99, 2567.

Stribling, T., Matthaeus, W. H. and Oughton, S. 1995 Magnetic helicity in magnetohydrodynamic turbulence with a mean magnetic field. Phys. Plasmas 2, 1437-1452.

Tu, C.-Y. and Marsch, E. 1995 MHD Structures, Waves and Turbulence in the Solar Wind. Kluwer, Dordrecht.

Zank, G. P. and Matthaeus, W. H. 1992 The equations of reduced magnetohydrodynamics. J. Plasma Phys. 48, 85. 